\title{
Combined perforation of the rectum and urinary bladder secondary to autoanal stimulation: Report of a case
}

\author{
MARC S. TUCKER, D.O. \\ PETER SINAIKO, M.D. \\ Langhorne, Pennsylvania \\ LOU GALLO, D.O. \\ Stratford, New Jersey
}

A unique case of combined rectal and urinary bladder perforation secondary to autoanal stimulation via a wooden toilet plunger handle is reported. Previously published principles for repair of isolated cases of rectal or bladder trauma were combined to effect repair. With successful surgical procedures and appropriate follow-up care, the patient made an uneventful recovery. The current literature on these types of injuries is also reviewed.

Management strategies for this type of perforation include the following: (1) A high index of suspicion for this kind of injury should be maintained,

particularly when the patient is a man aged 20-40 years who presents with abdominal pain, absent bowel sounds, tenderness, guarding, varying degrees of rigidity, and rectal bleeding; (2) a history of rectal sexual activity is necessary, and physical examination must include rectal examination, with attention given to mucosal integrity; (3) early diagnosis and surgical repair is mandatory to avoid the morbidity and mortality associated with perforating injuries to the rectum or bladder; and (4) pre- and post-operative three-drug antibiotic therapy and tetanus immunoprophylaxis is encouraged.

During the past several years, there has been an increase in the number of reports ${ }^{1-5}$ of rectal per- foration secondary to accidents involving anal intercourse, including heterosexual, homosexual, and autostimulatory behavior. There have also been reports $s^{6,7}$ of perforating urinary bladder trauma, although this injury usually is secondary to motor vehicle accidents or violent activity.

In this paper, a unique case of combined penetrating rectal and bladder injury secondary to autoanal insertion of a wooden handle from a toilet plunger is presented. The current literature regarding the handling of similar types of injuries (combined injuries are very rarely reported) is reviewed. Also, the various kinds of morbidity associated with such trauma are discussed.

\section{Report of case}

A 29-year-old white man presented to the emergency room with diffuse abdominal pain and rectal bleeding. On questioning, the patient admitted to autorectal insertion of a wooden toilet plunger handle approximately 3 feet in length. He stated that he had been using the plunger to perform autoanal intercourse in the shower when he had slipped, and had felt the handle "pop" his rectum. At this instance he noticed rectal bleeding. The patient's medical and surgical history was unremarkable. He denied any use of medication or allergies.

Physical examination revealed a well-developed man in extremis. The patient was hemodynamically stable, with a blood pressure of $140 / 88 \mathrm{~mm}$. $\mathrm{Hg}$, heart rate of 92 beats per minute, and temperature of $97.4 \mathrm{~F}$. The HEENT examination was unremarkable, the lungs were clear to auscultation and percussion, and the heart had a regular rate and rhythm, without any murmurs, rubs, or gallops. Abdominal examination revealed decreased bowel sounds and slight distention. Diffuse abdominal tenderness, with guarding and rebound tenderness, was noted. Rectal examination revealed gross bleeding and a $3-\mathrm{cm}$. 
gen, creatinine, prothrombin, partial thromboplastin, and liver function study results were all within normal limits. Urinalysis revealed gross blood, with red blood cells too numerous to count.

Cystography revealed extra- and intra-peritoneal extravasation of contrast material and possible traumatic rectovesical fistula.

Preoperatively, a three-drug intravenous antibiotic regimen, including ampicillin (1 gm.), gentamicin (100 $\mathrm{mg}$.), and clindamycin (600 mg.), was started. Blood and urine culture and sensitivity specimens were obtained. Nasogastric suction was maintained throughout the presurgical period.

Exploratory laparotomy revealed a large perforation in the posterior aspect and dome of the urinary bladder. This was repaired by two-layer closure using chromic gut suture. Two suprapubic tubes were placed for postoperative drainage. A transverse loop colostomy was then performed, and the abdominal portion of the surgery was completed. The distal segment of the loop colostomy was not irrigated at the time of surgery. Under anoscopic guidance, the rectal perforation was closed in two-layer fashion with chromic gut suture.

The patient's postoperative course was uneventful, and he was tolerating a normal diet by the fifth day after surgery. On the seventh postoperative day, the bladder repair was reexamined by cystography, which showed no extravasation of dye. The suprapubic tubes were removed at this time. The patient received a 7-day course of triple antibiotic therapy, administered intravenously.

The patient was discharged on the eighth postsurgical day. He was examined 6 weeks later with sigmoidoscopy, and the rectal perforation had healed completely. The patient had closure of his transverse loop colostomy performed 2 months after the initial injury. With follow-up care, he is experiencing no further problems.

\section{Discussion}

Isolated cases have been reported in which the rectum was perforated secondary to manual-anal intercourse $e^{1,8}$ or where there was insertion of various objects into the rectum that lacerated, perforated, or were a challenge to remove. ${ }^{2-5,9}$ These injuries were described and treated according to the severity of the laceration and whether peritonitis was present.

Most of the experience in repairing colorectal perforation was obtained during the Vietnam war. ${ }^{10}$ In a 1977 article,${ }^{11}$ four approaches to repairing colon perforation were described, as follows: (1) simple repair and proximal diversion; (2) primary closure or resection and anastomosis of the wound with exteriorization; (3) formation of a double-barreled colostomy; and (4) Hartmann's procedure. Allen ${ }^{12}$ and Levanson and Cohen ${ }^{13}$ have detailed direct approaches, with repair of the rectal laceration and proximal diversion. Gatzen and associates $^{14}$ described a Miles resection for injuries that also devascularize the rectum.
The literature ${ }^{6,7,15}$ produced during the past few years on treatment of urinary bladder perforation supports primary repair, with two-layer closure and drainage for 1 to 2 weeks. This type of repair is indicated whether the trauma is penetrating or blunt.

In the present case, the principles presented in the literature were combined into a single procedure to effect repair. Also, as had been advocated in some articles, sigmoidoscopic examination was performed prior to reclosure of the colostomy to assure that there had been good healing.

The morbidity and mortality associated with perforating injuries to the rectum or bladder are due to delay in diagnosis (24 hours or longer) or related injuries. When a genitourologic injury is associated with a perforating rectal injury, the morbidity is significantly greater.

In a study by Allen, ${ }^{12}$ complications were encountered in approximately 40 percent of initial procedures and 37 percent of secondary procedures (colostomy closure). Intra-abdominal abscess was encountered most frequently; the incidence was much higher when there was a delay in diagnosis. Bacteremia, fistula formation, and iatrogenic complications during surgery, as well as wound infection and abscess, also occurred.

Allen ${ }^{12}$ also reported an increase in morbidity when loop colostomy was performed in lieu of divided colostomy. Complications included colocutaneous fistula, pelvic abscess, retroperitoneal abscess, osteomyelitis, peritonitis, bowel obstruction, and delayed pelvic hemorrhage. Again, these complications were reported in as high as 37 percent of the patient population. To decrease the complication rate from loop colostomy, some authors ${ }^{12,13}$ advocate copious irrigation of the distal segment at the time of surgery. When retained stool was present, there was an increase in colocutaneous fistula formation. There are also some Clostridium tetani in feces, and it has been postulated ${ }^{8}$ that fecal spillage may be the etiology of clinical tetanus. Therefore, tetanus immunoprophylaxis is recommended for all patients with this type of trauma, as was done in this case.

\section{Summary}

Combined perforation of the rectum and urinary bladder secondary to autoanal stimulation is rare. Aggressive management is essential because of the high morbidity and mortality associated with such injuries.

1. Reiner, S.C.: Colorectal laceration after manual-anal intercourse. Ann Emerg Med 13:130-2, Feb 84 
2. Crass, R.A., et al.: Colorectal foreign bodies and perforation. Am J Surg 142:85-8, Jul 81

3. Robertson, H.D., et al.: Management of rectal trauma. Surg Gynecol Obstet 154:161-4, Feb 82

4. Wiener, I., Rojas, P., and Wolma, F.J.: Traumatic colon perforation. Am J Surg 142:717-20, Dec 81

5. Witz, M., et al.: Anal erotic instrumentation. A surgical problem. Dis Colon Rectum 27:331-2, May 84

6. Carroll, P.R., and McAninch, J.W.: Major bladder trauma. Mechanisms of injury and a unified method of repair. J Urol 132:254-7, Aug 84

7. Hayes, E.E., Sandler, C.M., and Corriere, J.M.: Management of the ruptured bladder secondary to blunt abdominal trauma. J Urol 129:946-8, May 83

8. Brenner, B.E., and Simon, R.R.: Anorectal emergencies. Ann Emer Med 12:367-76, Jun 83

9. Barone, J., Yee, J., and Nealon, T.F., Jr.: Management of foreign bodies and trauma of the rectum. Surg Gynecol Obstet 156:453-7, Apr 83

10. Halvorsen, J.F., Vagn Nielsen, O., and Bugge-Asperheim, B.: Injuries of the colon and rectum. Ann Chir Gynaecol 70:287-91, 1981

11. Sohn, N., Weinstein, M.A., and Gonchar, J.: Social Injuries of the rectum. Am J Surg 134:611-2, Nov 77

12. Allen, B.D.: Penetrating wounds of the rectum. Texas Med 69:77-81, 1969
13. Levanson, G.S., and Cohen, A.: Management of rectal injuries. Am J Surg 122:226-30, Aug 71

14. Getzen, L.C., Pollak, E.W., and Wolfman, E.F., Jr:: Abdominoperineal resection in the treatment of devascularizing rectal injuries. Surgery $82: 310-3$, Sep 77

15. Koskela, E., et al.: Subcutaneous rupture of the urinary bladder. Ann Chir Gynaecol 66:150-3, 1977

Accepted for publication in February 1986. Updating, as necessary, has been done by the authors.

Dr. Tucker is a resident in general surgery at Delaware Valley Medical Center, Langhorne, Pennsylvania; Leonard Segal, D.O., FACOS, is resident trainer. Dr. Sinaiko is an attending physician in the Department of Urology at Delaware Valley Medical Center. Dr. Gallo is a professor of surgery at the University of Medicine and Dentistry of New Jersey-School of Osteopathic Medicine, Camden, New Jersey.

Dr. Tucker, 75 Pine Glen Road, Langhorne, Pennsylvania 19047. 